\title{
LXXIII. Does motion through the Æther cause double refraction?
}

\section{Lord Rayleigh O.M. F.R.S.}

To cite this article: Lord Rayleigh O.M. F.R.S. (1902) LXXIII. Does motion through the Æther cause double refraction? , Philosophical Magazine Series 6, 4:24, 678-683, DOI: 10.1080/14786440209462891

To link to this article: http://dx.doi.org/10.1080/14786440209462891

曲 Published online: 15 Apr 2009.

Submit your article to this journal $[\pi$

Џ Article views: 9

Q View related articles $\square$

Citing articles: 10 View citing articles 5 
metals, namely, about $2.5 \times 10^{-6} \mathrm{~cm}$. for the upper limit. The experiments on platinum films, however, would indicate that for platinum films the thickness of the layer is much less. According to the corpuscular theory this transition-layer would be determined by $\lambda$, the mean free path of the corpuscles, and $\lambda$ has about the same value for a number of the metals*.

The thickness at which the specific resistance of the platinum film becomes abnormal cannot be taken as the value of $\lambda$ in the metal itself, owing to the properties of the films being so very different from those of the metal.

Summary of Results.

(i.) The specific resistance of the films deposited in racuo by the cathode discharge is several times greater than the specific resistance of the metal from which they are deposited.

(ii.) The specific resistance of platinum films which have been subjected to the same treatment remains constant above a thickness of about $7 \times 10^{-7} \mathrm{~cm}$. Below this thickness the increase of specific resistance with decrease in thickness is very rapid.

(iii.) Heat decreases the resistance of both silver and platinum films, and the thinner the film the greater the decrease. In platinum films the greatest decrease is produced by the electric current.

(iv.) The values obtained for $\lambda$, the mean free path of the corpuscle in the metal, are of the same order as those obtained from the change of resistance produced by a transverse magnetic field.

In conclusion my most sincere thanks are due to Professor Thomson for his valuable advice and kindly interest throughout the whole course of the investigation described in this paper.

Cavendish Laboratory, May 12, 1902.

LXXIII. Does Motion through the AEther cause Double Refraction? By Lord RAYLeIGH, O.M., F.R.S.†

rTHE well-known negative result of the Michelson-Morley experiment in which interference takes place between two rays, one travelling to and fro in the direction of the earth's motion, and the other to and fro in a perpendicular direction, is most naturally interpreted as proving that the

* Phil. Mag. June 1902, p. 655.

+ Communicated by the Author. Read before Section A of the British Association at Belfast. 
ather in the laboratory shares the earth's motion. But other phenomena, especially stellar aberration, favour the opposite theory of a stationary rether. The difficulty thus arising has been met by the at first sight startling hypothesis of FitzGerald and Lorentz that solid bodies, such as the stone platform of Michelson's apparatus, alter their relative dimensions, when rotated, in such a way as to compensate the optical clange that might naturally be looked for. Larmor (" Ather and Matter,' Cambridge, 1900) has shown that al good case may be made out for this view.

It occurred to me that such a deformation of matter when moving through the rther might be accompanied by a sensiblo double refraction ; and as the beginning of double refraction can be tested with extraordinary delicacy, I thought that even a small chance of arriving at a positive result justified a careful experiment. Whether the result were positive or negative, it might at least afford further guidance for speculation upon this important and delicate subject.

So far as liquids are concerned, the experiment is of no great difficulty, and the conclusion may be stated that there is no double refraction of the order to be expected, that is comparable with $10^{-8}$ of the single refraction*. But the question arises whether experiments upon liquids really settle the matter. Probably no complete answer can be given, unless in the light of some particular theory of these relations. But it may be remarked that the liquid condition is no obstacle to the development of double refraction under electric stress, as is shown in Dr. Kerr's experiments.

The apparatus was mounted upon the same revolving hoard as was employed for somewhat analogous experiment: noon the rotation in quartz (Phil. Mag. vol. iv. p. 215, 1902). Light, at first from the electric are but later and preferably from lime heated by an oxyhydrogen jet, after pasing a spectacle-lens;o held as to form an image of the source upon the analysing nicol, was polarized by the tirst nicol in a plane inclined to the horizontal at $45^{\circ}$. The liquid, held in a horizontal tube closed at the ends by plates of thin glass, was placed, of course, between the nicols. When at 12 o'clock the board stands north and south, the earth's motion is transverse and the situation is such as to exhibit any double refraction which may ensue. It might be supposed, for instance, that luminous vilurations parallel to the earth's motion, $i$. e. east and west, are propagated a little differently from those whose direction is transverse to the earth's motion, $i . e$. vertical. But if the

$*^{*} 10^{-3}=\left(10^{-4}\right)^{2}$, where $10^{-4}$ is the ratio of the velocity of the earth in its orbit to the velocity of light. 
board be turned through a right angle so as to point east and west, both directions of vibration for light passing the tube are transverse to the earth's motion, and therefore no double refraction could manifest itself. The question is whether: turning the board from the north and south position to the east and west position makes any difference. In no case is any effect to be expected from a rotation through $180^{\circ}$, and such effect as a rotation through $90^{\circ}$ may entail must be of the second order in the ratio which expresses the velocity of the earth relatively to that of light.

It should not be overlooked that according to the theory of a stationary ather, we have to do not only with the motion of the earth in its orbit, but also with that of the sun in space. The latter is supposed to be much the smaller, and to be directed towards the constellation Hercules. In the month of April, when successful experiments were first made, the two motions would approximately conspire.

If the suggested double refraction, due to the earth's motion, were large enough, it would suffice to set the analysing nicol to extinction in one position of the board, and to observe the revival of light consequent on a rotation of the latter throngh $90^{\circ}$. But a more delicate method is possible and necessary. Between the polarizing nicol and the liquid column we introduce a strip of glass whose length is horizontal and transverse to the board. This strip, being supported (at two points) near the middle of its length, and being somewhat loaded at its ends, is in a condition of strain, and causes: a revival of light except in the neighbourhood of a horizontal band along the "neutral axis." Above and below this band the strained condition of the glass produces just such a double refraction as might be cansed by the motion of the liquid through the rether, so that the existence of the latter would be evidenced by a displacement of the dark band upwards or downwards. In order the better to observe a displacement, two horizontal wires are disposed close to th. bent glass so as just to inclose the band, and a small operaglass focussed upon these is introduced beyond the analysing nicol. The slightest motion of the band is rendered evident by changes in the feeble iliumination just inside the wires.

The board is mounted upon a point so as to revolve with the utmost freedom. The point is carried on the table and faces upwards. The bearing is a small depression in an iron strap, rigidly attached to the board, and raised sufticiently to give stability. The gas-leading tubes are connected in such a manner as to give rise to no forces which could appreciably vary as the board turns. 
Observations were made upon bisulphide of carbon in a tube $76 \mathrm{cms}$. long, and upon water in a tube $73 \frac{1}{2} \mathrm{cms}$. long. In neither case could the slightest shift of the band be seen on rotation of the board from the north-south position to the east-west position, whether at noon or at 6 P.M. The time required to pass from one observation to the other did not exceed 15 seconds, and the alternate observations were repeated until it was quite certain that nothing could be detected.

Of course the significanee of this result depends entirely noron the delicacy of the apparatus, and it is worth little without an estimate of the smallest double refraction that would have been detected. It may eren be objected that the investigation stands self-condemned. In consequence of the earth's magnetism there must be a rotation of the plane of polarization when the light traverses the bisulphide of carbon in the north and south position; and this effect, it may be argued, ought to manifest itself upon rotation of the board.

To take the objection first, it is easy to calculate the rotation of the plane of polarization. For one c.G.s. unit of magnetic potential the rotation in $\mathrm{CS}_{2}$ at $18^{\circ}$ is $\cdot 042$ minute of angle*. In the present case the length is 76 cms. and the earth's horizontal furce is 18 ; so that the whole rotation to be expected $\uparrow$ is

$$
76 \times \cdot 18 \times \cdot 042=\cdot 58
$$

So small a rotation of the plane, which would show itself, if at all, by a fading and not by a displacement of the band, is below the limit of observation.

The delicacy of the apparatus for its purpose may, indeed, be inferred indirectly from the rotation of the nicol found necessary to engender a marked revival of light at the darkest part of the band. If $\theta$ be this angle, the revived light is $\sin ^{2} \theta$, expressed as a fraction of the maximum obtainable with parallel nicols. In the actual observation the nicols remain accurately crossed, and the question is as to the effect of a double refiaction causing e.g. a retardation of vertical vibrations relatively to horizontal ones. If this retardation amounted to $\frac{1}{2} \dot{\lambda}, \lambda$ being the wave-length, the effect would be the same as of a rotation of the nicol through $90^{\circ}$. In general, a retardation of phase $\epsilon$, in place of $\pi$, gives a revival of light measured by $\sin ^{2}\left(\frac{1}{2} \epsilon\right)$. If the revivals of light in the two cases be the same, we may equate

* Phil. Trans. clxxvi. j. 343 (1885) ; Scientific Papers, vol. ii. p 377.

+ The difference between astronomical and magnetic north is here neglected. 
$\theta$ to $\frac{1}{2} \epsilon$. Hence if we find that rotation $\theta$ produces a sensible effect in lessening the darkness at the darkest place, we may infer that there is delicacy sufficient to detect a relative retardation of $2 \theta$ due to double refraction. This comparison would apply if the test for double refraction were made by simple observation of the revival of light. As actually carried out by location of the band, the test must be many times more delicate.

It was found that a marked fading of the band attended a rotation of the nicol through $4^{\prime}$. According to this $\epsilon$ would be $\frac{1}{450}$; or since a retardation of $\frac{1}{2} \lambda$ corresponds to $\epsilon=\pi$, a retardation amounting to $\frac{1}{1400} \times \frac{1}{2} \lambda$ should be perceptible many times over, regard being paid to the superior delicacy of the method in which a band is displaced relatively to fixed marks.

Another and perhaps more satisfactory method of determining the sensitiveness was by introducing a thin upright strip of glass which could be compressed in the direction of its length by small loads. These loads were applied symmetrically in such a manner as to cause no flexure. The double refraction due to the loads is of exactly the character to be tested for, and accordingly this method affords a very direct check. If the load be given, the effect is independent of the length of the strip and of its thickness along the line of vision, but is inversely as the width. 'The strip actually employed had a width of $15 \mathrm{~mm}$.; and the application (or removal) of a total of $50 \mathrm{gms}$. caused a marked shifting of the band, while 25 gms. was just perceptible with certainty.

To interpret this we may employ some results of Wertheim (Mascart's Traité d'Optique, t. ii. p. 232), who found that it requires a load of 10 kilograms per millimetre of width to give a relative retardation of $\frac{1}{2} \lambda$, so that with the actnal strip the load would need to be 150 kilograms. The retardation just perceptible is accordingly $\frac{1}{2} \lambda \div 6000$. This may be considered to agree well with what was expected from the effect of rotating the nicol.

We have now only to compare the relative retardation which would be detected with the whole retardation incurred in traversing the $76 \mathrm{~cm}$. of bisulphide of carbon. In this length there are contained 1,200,000 wave-lengths of yellow light, or 2,400,000 half wave-lengths. The retardation due to the refraction may be reckoned at ' $t \dot{t}$ of this, or $1,440,000$ half wave-lengths. Thus the double refraction that might be detected, estimated as a fraction of the whole refraction, 
is $1 \cdot 2 \times 10^{-10}$. The effect to be expected is of the order $10^{-8}$, so that there is nearly 100 times to spare. The above relates to the bisulphide of carbon. With the water the delicacy of the test was somewhat less.

When it is attempted to replace the liquid by solid matter, the difficulties of experiment are greatly increased. The hest results that I have been able to obtain were with built up thicknesses of plate-glass. A sufficient thickness in one piece is liable to exhilit too much double refraction from the effect of internal strains. A number of triangular pieces of plate-glass, no larger than necessary, and about $6 \mathrm{~mm}$. thick, were put together in a trough to a total thickness of about $110 \mathrm{~mm}$. The interstices between the faces being filled up with bisulphide of carbon, the internal reflexions were sufficiently reduced. One difficulty is to get quit of motes and threads which adhere to the glass and become extraordinarily conspicuous. Advantage was thought to be derived from shaking up the bisulphide of carbon with strong sulphuric acid. At the best the residual motes and specks in the glass interfere very seriously with the observation, and the loss of light due to imperfect transparency operates in the same direction. The least load upon the upright strip that could be detected with certainty was now 100 grms., so that as compared with the observations upon liquid there was a loss of delicacy of four times. In addition to this, the effect to be expected is reduced in the proportion of $\tau: 1$, that being the ratio of lengths traversed by the light. Thus in all we lose $2 \varnothing$ times as compared with the liquid. In the latter case we calculated a margin of 100 times, so that here there would remain a margin of about 3 times.

A subsequent attempt was made to increase the total thickness of the combined glasses to about $220 \mathrm{~mm}$., but no real advantage was gained. The loss of light and increase of disturbance from motes and residual double refraction prejudiced the delicacy in about the same proportion ats the length of path was increased.

But although the results of the observations upon solids are very much less satisfactory than in the case of liquids, enough remains to justify us in concluding that even here there is no double refraction (of the order to be expected) due to motion through the ather.

Terling Place, Witham. 